\title{
A NOTE ON MEAN SQUARE MAXIMUM AND MINIMUM PRINCIPLES IN DYNAMIC LINEAR VISCOELASTICITY*
}

\author{
BY \\ W. A. DAY \\ Hertford College, Oxford
}

\begin{abstract}
The purpose of this note is to point out that, in certain circumstances, the mean square values of the displacement, the strain, and the stress in a viscoelastic slab which is undergoing steady trigonometric (but not necessarily periodic) oscillation satisfy maximum or minimum principles.
\end{abstract}

We consider an isotropic and homogeneous slab which occupies the region $0 \leqslant x \leqslant l$, where $x$ is a cartesian coordinate, and we suppose the displacement to be parallel to the $x$-axis at all times $t$. Then $u(x, t)$, the $x$-component of the displacement, and $e(x, t)$ and $s(x, t)$, which are, respectively, the $x x$-components of strain and stress, satisfy the strain-displacement equation

$$
e=\partial u / \partial x
$$

the Boltzmann law

$$
s(x, t)=g(0) e(x, t)+\int_{-\infty}^{t} g^{\prime}(t-\tau) e(x, \tau) d \tau,
$$

and the equation of motion

$$
\partial s / \partial x=\rho \partial^{2} u / \partial t^{2}
$$

$\rho$ being the mass density.

We shall restrict the relaxation function $g$ by requiring that its derivative $g^{\prime}$ be absolutely integrable on $0 \leqslant \tau<\infty$ and that

$$
\int_{0}^{\infty}\left|g^{\prime}(\tau)\right| d \tau<g(0)
$$

These restrictions being granted, the equilibrium elastic modulus

$$
g(\infty)=\lim _{\tau \rightarrow \infty} g(\tau)
$$

exists and is positive, and we can define the complex modulus $K(\omega)$ and the phase lag

\footnotetext{
* Received July 12, 1983.
} 
$\phi(\omega)$ in the usual ways, namely

$$
\begin{aligned}
& K(\omega)=g(0)+\int_{0}^{\infty} g^{\prime}(\tau) \exp (-i \tau \omega) d \tau, \\
& \phi(\omega)=\arg K(\omega), \quad 0 \leqslant \phi(\omega)<2 \pi .
\end{aligned}
$$

We notice that $K(0)=g(\infty)$ and that $\phi(0)=0$.

It is not really necessary for us to invoke quite so restrictive a constitutive relation as the Boltzmann law; we could replace it by a more general linear hereditary law of the kind envisaged by König and Meixner, by Gurtin and Sternberg, and by Leitman and Mizel. (For references to the works of these authors and for an account of linear hereditary laws see Sec. 6 of Leitman and Fisher's article [1].) We shall not carry through this generalization ourselves because it will be readily apparent to the reader what modifications would have to be made to our argument.

Definition. The ordered triple $\{u, e, s\}$ is said to be a trigonometric viscoelastic process if (i) $u, e, s$ can be expressed as real trigonometric sums

$$
\begin{aligned}
& u(x, t)=\operatorname{Re} \sum U(x, \omega) \exp (i \omega t), \\
& e(x, t)=\operatorname{Re} \sum E(x, \omega) \exp (i \omega t), \\
& s(x, t)=\operatorname{Re} \sum S(x, \omega) \exp (i \omega t),
\end{aligned}
$$

each sum being taken over the same finite set of positive Fourier exponents $\omega$, and (ii) the sums satisfy equations (1), (2), (3) for $0 \leqslant x \leqslant l$ and $-\infty<t<\infty$.

It follows that, if $\{u, e, s\}$ is a trigonometric viscoelastic process, the complex-valued functions $U, E, S$ are related through the equations

$$
\begin{aligned}
E & =d U / d x, \\
S & =K E, \\
d S / d x & =-\rho \omega^{2} U .
\end{aligned}
$$

Our aim is to examine the dependence upon $x$ of the mean square displacement

$$
M(x ; u)=\lim _{T \rightarrow \infty} \frac{1}{2 T} \int_{-T}^{T} u(x, t)^{2} d t,
$$

the mean square strain

$$
M(x ; e)=\lim _{T \rightarrow \infty} \frac{1}{2 T} \int_{-T}^{T} e(x, t)^{2} d t,
$$

and the mean square stress

$$
M(x ; s)=\lim _{T \rightarrow \infty} \frac{1}{2 T} \int_{-T}^{T} s(x, t)^{2} d t .
$$

The reader will notice that these mean square values have been defined in just the same way as in the theory of almost periodic functions and as in Wiener's theory of generalized harmonic analysis [2, Chapter IV]. It is very well known that the limits involved in the 
definitions always exist and that their values are

$$
\begin{aligned}
& M(x ; u)=\frac{1}{2} \sum|U(x, \omega)|^{2}, \\
& M(x ; e)=\frac{1}{2} \sum|E(x, \omega)|^{2}, \\
& M(x ; s)=\frac{1}{2} \sum|S(x, \omega)|^{2} .
\end{aligned}
$$

We shall find that, provided the exponents $\omega$ are sufficiently small, we can prove maximum or minimum principles in two important cases, namely when one of the faces is clamped or when one of the faces is stress-free. In either case, of course, the trigonometric viscoelastic process has to be sustained by supplying an appropriate displacement or stress on the other face.

In order to make clear how small the exponents have to be we introduce the real numbers

$$
\begin{aligned}
& \xi(\omega)=2 \omega \sqrt{\frac{\rho}{|K(\omega)|}} \cdot \sin \frac{1}{2} \phi(\omega), \\
& \eta(\omega)=2 \omega \sqrt{\frac{\rho}{|K(\omega)|}} \cdot \cos \frac{1}{2} \phi(\omega),
\end{aligned}
$$

in terms of which the required conditions are:

(*) The exponents $\omega$ satisfy

$$
\begin{gathered}
|\eta(\omega)| l<\pi, \\
|\xi(\omega)| \sinh (|\xi(\omega)| l)<|\eta(\omega)| \sin (|\eta(\omega)| l) .
\end{gathered}
$$

Since

$$
\xi(\omega)=o(\omega) \text { and } \eta(\omega) \sim 2 \omega \sqrt{\frac{\rho}{g(\infty)}} \quad \text { as } \omega \rightarrow 0 \text {, }
$$

these conditions certainly hold if $\omega$ is sufficiently small.

If the material is elastic, with elastic modulus $\lambda$, that is if $g(\tau)=\lambda(0 \leqslant \tau<\infty)$, we have $\xi(\omega)=0$ and $\eta(\omega)=2 \omega \sqrt{\rho / \lambda}$ and, therefore, $(*)$ holds if and only if $\omega<\omega_{0}$, where $\omega_{0}=(\pi / 2 l) \sqrt{\lambda / \rho}$ is the least of the natural exponents associated with the free vibration problem

$$
\lambda \frac{\partial^{2} u}{\partial x^{2}}=\rho \frac{\partial^{2} u}{\partial t^{2}}, \quad u(0, t)=\frac{\partial u}{\partial x}(l, t)=0 .
$$

We record a fact to be used later, namely that

$$
\frac{1}{2}(\xi+i \eta)=i \omega \sqrt{\frac{\rho}{|K|}} \cdot \exp \left(-\frac{1}{2} i \phi\right)
$$

and, therefore, that

$$
\frac{1}{4}(\xi+i \eta)^{2}=-\frac{\rho \omega^{2}}{|K|} \exp (-i \phi)=-\frac{\rho \omega^{2}}{K}
$$


Theorem 1. Suppose that $\{u, e, s\}$ is a trigonometric viscoelastic process, that each exponent satisfies $(*)$, and that

$$
u(0, t)=0 \quad(-\infty<t<\infty) .
$$

Then $M(x ; u)$ increases, and $M(x ; e)$ and $M(x ; s)$ decrease, on $0 \leqslant x \leqslant l$. In particular, $M(x ; u)$ assumes its maximum at $x=l$, while $M(x ; e)$ and $M(x ; s)$ assume their maxima at $x=0$ and their minima at $x=l$.

TheOREM 2. Suppose that $\{u, e, s\}$ is a trigonometric viscoelastic process, that each exponent satisfies $(*)$, and that

$$
s(0, t)=0 \quad(-\infty<t<\infty) .
$$

Then $M(x ; u)$ decreases, and $M(x ; e)$ and $M(x ; s)$ increase, on $0 \leqslant x \leqslant l$. In particular, $M(x ; u)$ assumes its maximum at $x=0$ and its minimum at $x=l$, while $M(x ; e)$ and $M(x ; s)$ assume their maxima at $x=l$.

Each of these theorems depends upon the following remark.

LEMMA. If $a$ and $b$ are real numbers such that

$$
|b| l<\pi, \quad|a| \sinh (|a| l)<|b| \sin (|b| l)
$$

then $\cosh a x-\cos b x$ increases, and $\cosh a x+\cos b x$ decreases, on $0 \leqslant x \leqslant l$.

To verify that $\cosh a x-\cos b x$ increases we note that if $|b| l<\pi$ then $\sin (|b| x)>0$ for $0<x<l$ and, therefore,

$$
\begin{aligned}
\frac{d}{d x}(\cosh a x-\cos b x) & =a \sinh a x+b \sin b x \\
& =|a| \sinh (|a| x)+|b| \sin (|b| x) \\
& >0 \quad(0<x<l) .
\end{aligned}
$$

To verify that $\cosh a x+\cos b x$ decreases we use the fact that $\sinh (|a| x)$ is a convex function of $x$ for $x \geqslant 0$, while $\sin (|b| x)$ is a concave function of $x$ for $0 \leqslant x \leqslant l$ provided that $|b| l<\pi$. Thus, these functions satisfy the inequalities

$$
\sinh (|a| x)<\frac{x}{l} \sinh (|a| l) \quad \text { and } \sin (|b| x)>\frac{x}{l} \sin (|b| l) \quad(0<x<l)
$$

and, therefore,

$$
\begin{aligned}
\frac{d}{d x}(\cosh a x+\cos b x) & =a \sinh a x-b \sin b x \\
& =|a| \sinh (|a| x)-|b| \sin (|b| x) \\
& <\frac{x}{l}(|a| \sinh (|a| l)-|b| \sin (|b| l)) \\
& <0 \quad(0<x<l) .
\end{aligned}
$$

We are now in position to prove Thm. 1. On eliminating $E$ and $S$ between Eqs. (7), (8), (9) we see that $U$ is a solution of the differential equation

$$
\frac{d^{2} U}{d x^{2}}=-\frac{\rho \omega^{2}}{K} U=\frac{1}{4}(\xi+i \eta)^{2} U
$$

The hypothesis (10), to the effect that the face $x=0$ is clamped, tells us that $U(0, \omega)=0$ 
and, therefore, that $U$ must be a function of the form

$$
U=A \sinh \frac{1}{2}(\xi+i \eta) x,
$$

where $A=A(\omega)$ is complex. It follows that $E$ and $S$ are

$$
\begin{aligned}
& E=\frac{1}{2} A(\xi+i \eta) \cosh \frac{1}{2}(\xi+i \eta) x, \\
& S=\frac{1}{2} K A(\xi+i \eta) \cosh \frac{1}{2}(\xi+i \eta) x,
\end{aligned}
$$

and, since

$$
\begin{aligned}
& \left|\sinh \frac{1}{2}(\xi+i \eta) x\right|^{2}=\frac{1}{2}(\cosh \xi x-\cos \eta x), \\
& \left|\cosh \frac{1}{2}(\xi+i \eta) x\right|^{2}=\frac{1}{2}(\cosh \xi x+\cos \eta x),
\end{aligned}
$$

that the mean square values of the displacement, the strain, and the stress are

$$
\begin{aligned}
& M(x ; u)=\frac{1}{4} \sum|A(\omega)|^{2}(\cosh \xi(\omega) x-\cos \eta(\omega) x), \\
& M(x ; e)=\frac{1}{16} \sum|A(\omega)|^{2}\left(\xi(\omega)^{2}+\eta(\omega)^{2}\right)(\cosh \xi(\omega) x+\cos \eta(\omega) x), \\
& M(x ; s)=\frac{1}{16} \sum|K(\omega) A(\omega)|^{2}\left(\xi(\omega)^{2}+\eta(\omega)^{2}\right)(\cosh \xi(\omega) x+\cos \eta(\omega) x) .
\end{aligned}
$$

On appealing to the conditions (*) and to the lemma, with $a=\xi$ and $b=\eta$, we see that we have proved $\mathrm{Thm} .1$.

When we turn to Thm. 2, the hypothesis (11), to the effect that the face $x=0$ is stress-free, tells us that $S(0, \omega)=0$ and, therefore, that $d U(0, \omega) / d x=E(0, \omega)=0$. Thus $U, E$, and $S$ must be functions of the form

$$
\begin{aligned}
& U=A \cosh \frac{1}{2}(\xi+i \eta) x, \\
& E=\frac{1}{2} A(\xi+i \eta) \sinh \frac{1}{2}(\xi+i \eta) x, \\
& S=\frac{1}{2} K A(\xi+i \eta) \sinh \frac{1}{2}(\xi+i \eta) x,
\end{aligned}
$$

and the corresponding mean square values must be

$$
\begin{aligned}
& M(x ; u)=\frac{1}{4} \sum|A(\omega)|^{2}(\cosh \xi(\omega) x+\cos \eta(\omega) x), \\
& M(x ; e)=\frac{1}{16} \sum|A(\omega)|^{2}\left(\xi(\omega)^{2}+\eta(\omega)^{2}\right)(\cosh \xi(\omega) x-\cos \eta(\omega) x), \\
& M(x ; s)=\frac{1}{16} \sum|K(\omega) A(\omega)|^{2}\left(\xi(\omega)^{2}+\eta(\omega)^{2}\right)(\cosh \xi(\omega) x-\cos \eta(\omega) x) .
\end{aligned}
$$

Theorem 2 follows on appealing once again to the lemma.

\section{REFERENCES}

[1] M. J. Leitman and G. M. C. Fisher, The linear theory of viscoelasticity, in Encyclopedia of Physics, V1a/3, Springer-Verlag, Berlin, 1973

[2] N. Wiener, The Fourier integral and certain of its applications, Cambridge University Press, Cambridge, 1933 